\title{
Design of identification and printing module circuit for garbage collection system based on Internet of things technology
}

\author{
Qing Yan ${ }^{1 *}$, Lianqin $\mathrm{Jia}^{1}$, Qin $\mathrm{Liu}^{1}$, and Chengsheng Cui ${ }^{2}$ \\ ${ }^{1}$ Shandong Institute of commerce and technology, Jinan, Shandong,250103, China \\ ${ }^{2}$ Qingdao lugui Recycling Industry Development Co., Ltd, Qingdao Shandong,266000, China
}

\begin{abstract}
Under the premise of the implementation of waste classification in China, the public has designed a lot of garbage collection systems, but all of them have single use. Therefore, the author designs the identification and printing module, so as to expand the use of the garbage collection system.
\end{abstract}

\section{Introduction}

With the development of economy and the improvement of people's living standards, the output of domestic waste in China has shown an exponential growth rapidly. Domestic waste occupies land, pollutes the environment, and even affects human health, which has caused great attention of the country. Now the country is actively promoting the garbage classification, realizing the garbage classification treatment, and carrying out the garbage classification collection It has social, economic and ecological benefits to reduce the amount and equipment of garbage treatment, reduce the cost of treatment, minimize the pollution of garbage to the environment, reduce land erosion and reduce the consumption of land resources. At the same time, we should make full use of the existing production capacity and recycle recyclable products, which has social, economic and ecological benefits.

In this context, many garbage collection systems have been born. Through investigation, the author found that the existing garbage collection systems can be divided into the following two categories: one is a platform that collects garbage information and facilities information, transportation vehicle scheduling, garbage sorting and recycling, etc. For example: the residential garbage sorting and recycling system based on the Internet of things is based on RFID technology, making full use of the Internet of things technology, using advanced hardware equipment, perfect software management system and sound regulatory mechanism [1] [4]. The other is to realize the effective supervision of waste recovery equipment. For example, the intelligent supervision system of garbage collection equipment based on NB-LOT Technology: using NBLOT technology to realize intelligent monitoring and management of garbage collection equipment, timely inform the environmental sanitation staff of the detected information, and provide the best garbage cleaning route, improve the efficiency and quality of environmental sanitation, and accelerate the development process of smart city [2-3] [5].

\section{Detailed analysis of identification and printing module}

The above two types are focused on how to carry out the garbage removal and transportation. In fact, recycling recyclable products will produce great economic benefits. On this basis, we improved the garbage collection system, improved the identification and printing module on the existing basis, so as to recycle the recyclable products. After the infrared sensor is used, the reader will activate the code reader after sensing the person. The reader will read the QR code on the user's mobile phone or the $\mathrm{QR}$ code on the card After receiving the barcode recycling instruction from the IOT, the user can send the recycling information to the user after receiving the barcode recycling instruction, and then send it to the user after receiving the barcode recycling instruction The user information can be identified by scanning the label, and the recycler will transfer the money corresponding to the recyclable items into the user's account, so as to realize the recycling of the recyclable items.

The specific hardware core design is as follows:

\subsection{CPU module}

Here we use stm32f103ret6 chip to control the module through programming. The specific circuit connection is shown in Figure 1:

\footnotetext{
*Email:yanqing801101@163.com
} 


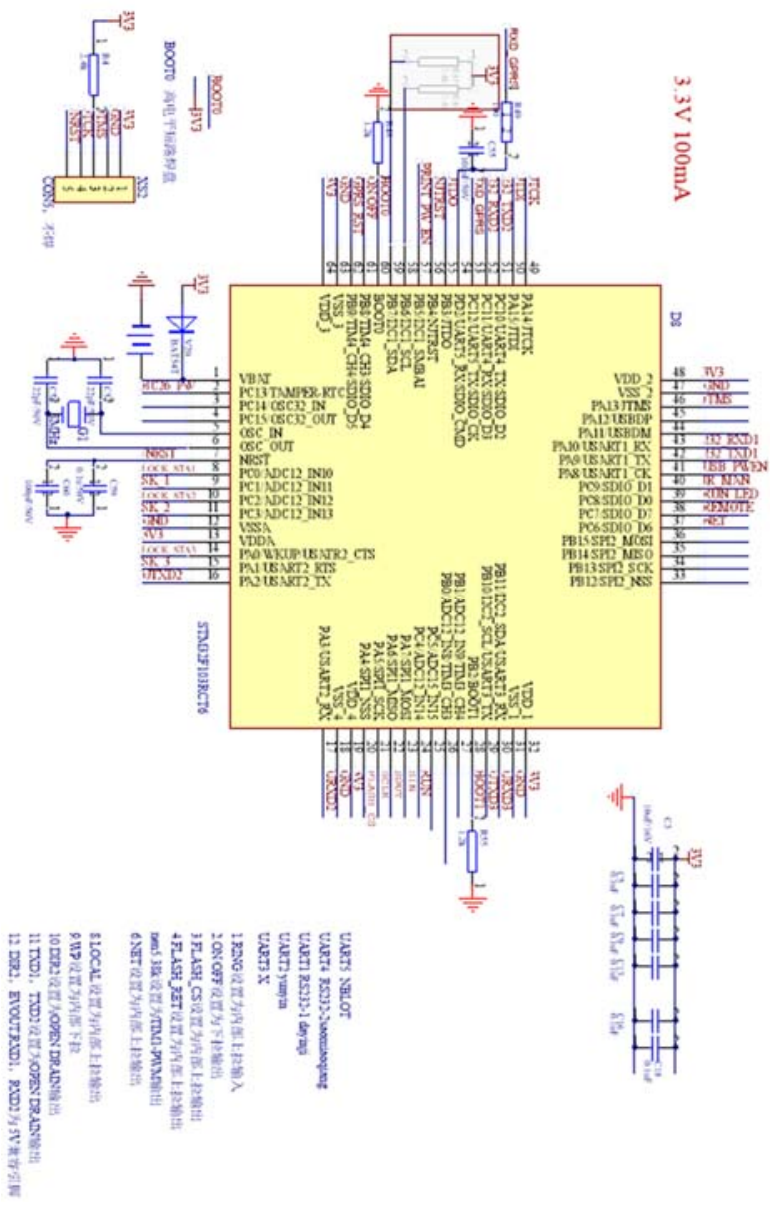

Fig.1 Circuit diagram of CPU

\subsection{NB-loT module}

The NB-IoT module uses bc26, which is a series of NBIoT modules with high performance and low power consumption. Through the NB-IoT radio communication protocol (3GPP rel.13 and 3GPP rel.14), bc26 module can establish communication with network operators' infrastructure. The default baud rate is $115200 \mathrm{bps}$. The specific circuit connection is shown in Figure 2:

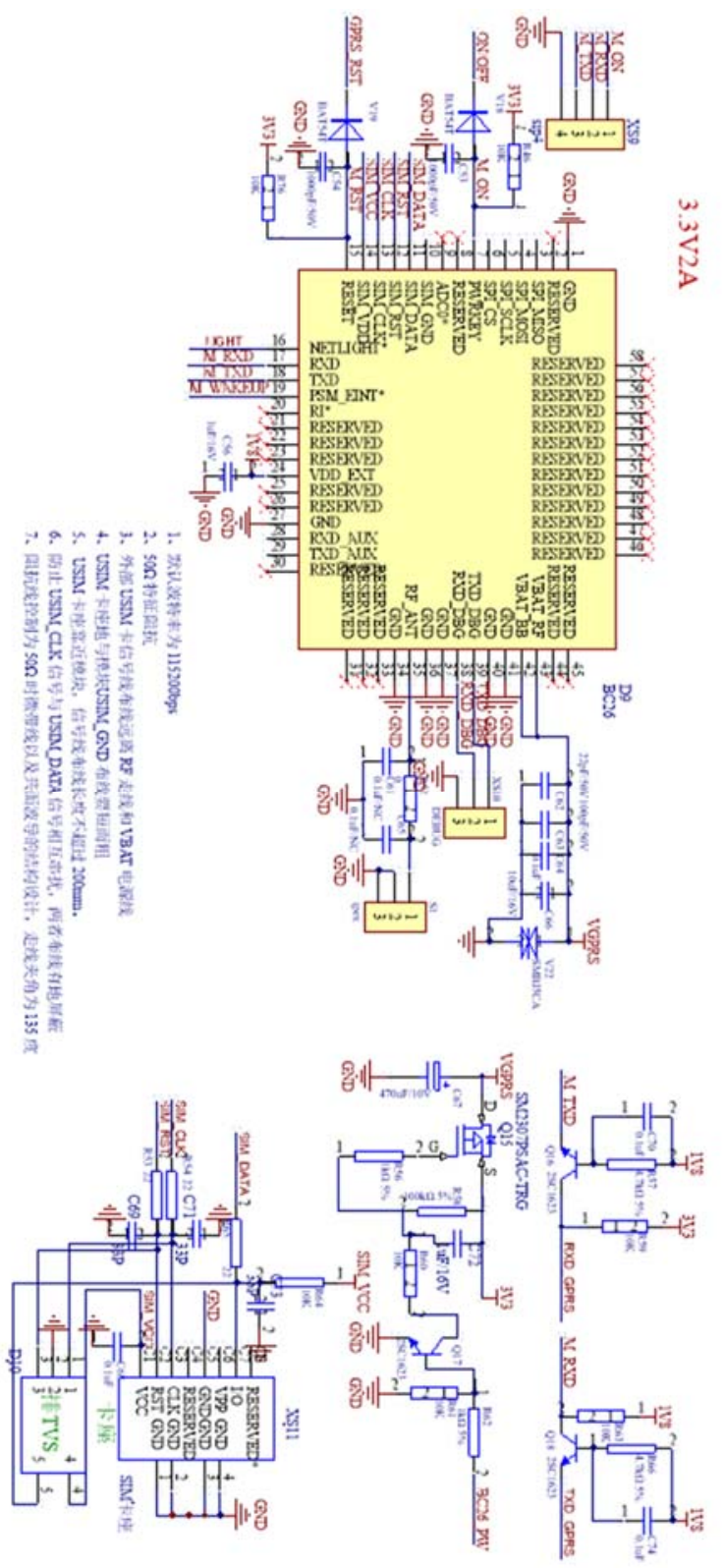

Fig. 2 Circuit diagram of $\mathrm{Nb}$ IOT

\subsection{2 communication module}

The UT3232 has two receivers and two drivers, and a dual charge-pump circuit. The device meets the requirements of TIA/EIA-232-F and provides the electrical interface between an asynchronous communication controller and the serial-port connector. The charge pump and four small external capacitors allow operation from a single $3.0 \mathrm{~V}$ to $5.5 \mathrm{~V}$ supply. The device operates at data signaling rates up to $250 \mathrm{kbit} / \mathrm{s}$ and a maximum of $35 \mathrm{~V} / \mathrm{us}$ driver output slew rate. The specific circuit connection is shown in Figure 3: 


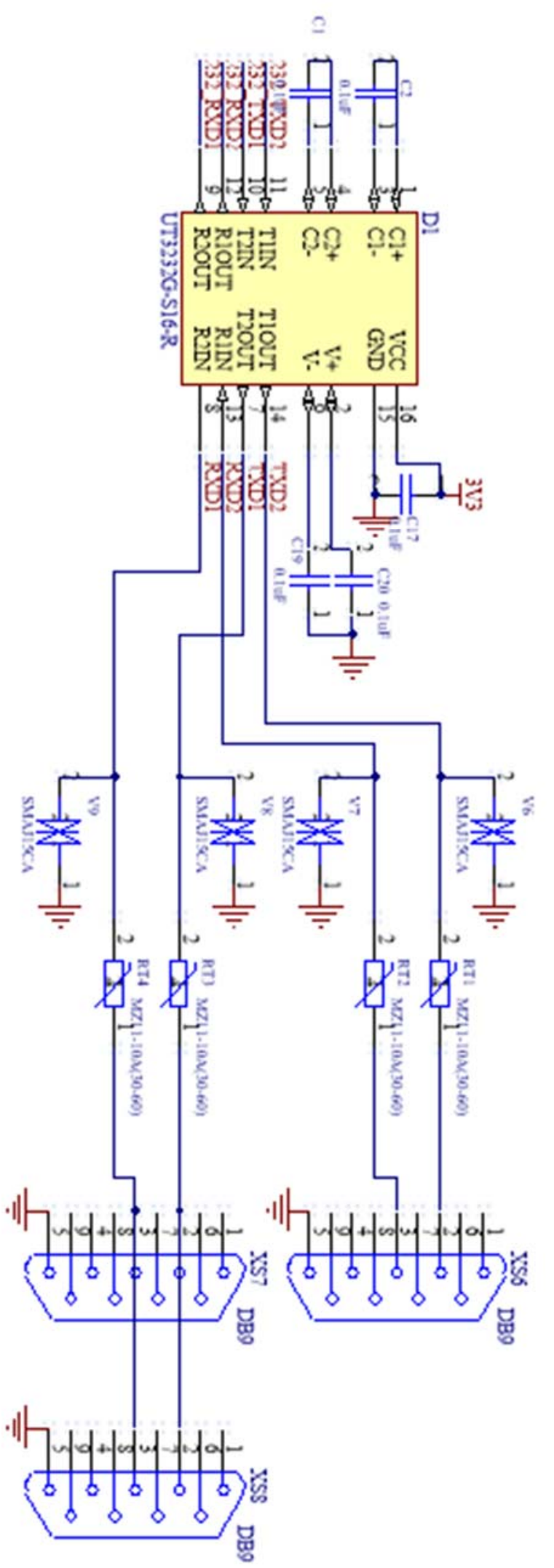

Fig.3 Circuit 232 communication of $\mathrm{Nb}$ IOT

\subsection{Lock control system}

The specific circuit connection is shown in Figure 4:
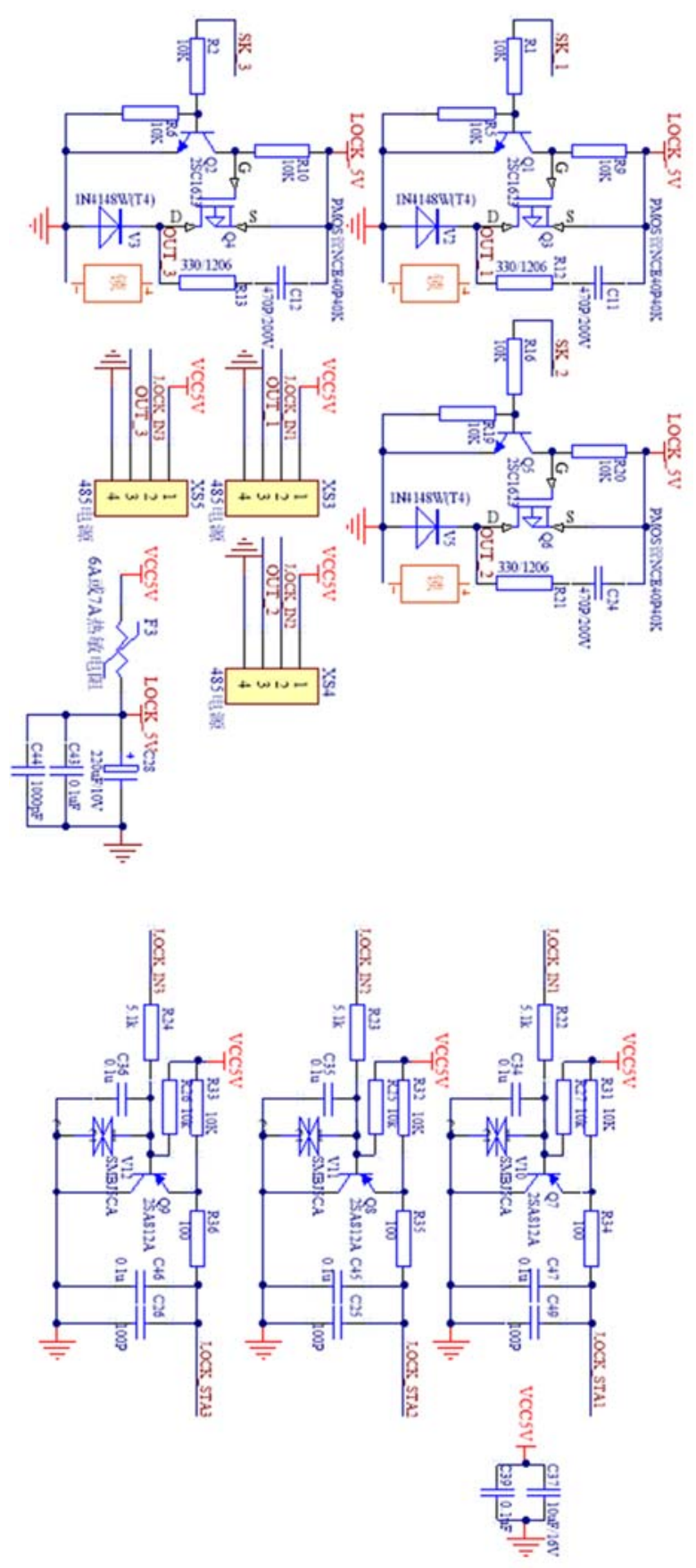

Fig.4 Circuit diagram of lock control part

\subsection{PCB diagram of the circuit}

The PCB diagram of the circuit is shown in Figure 5 : 


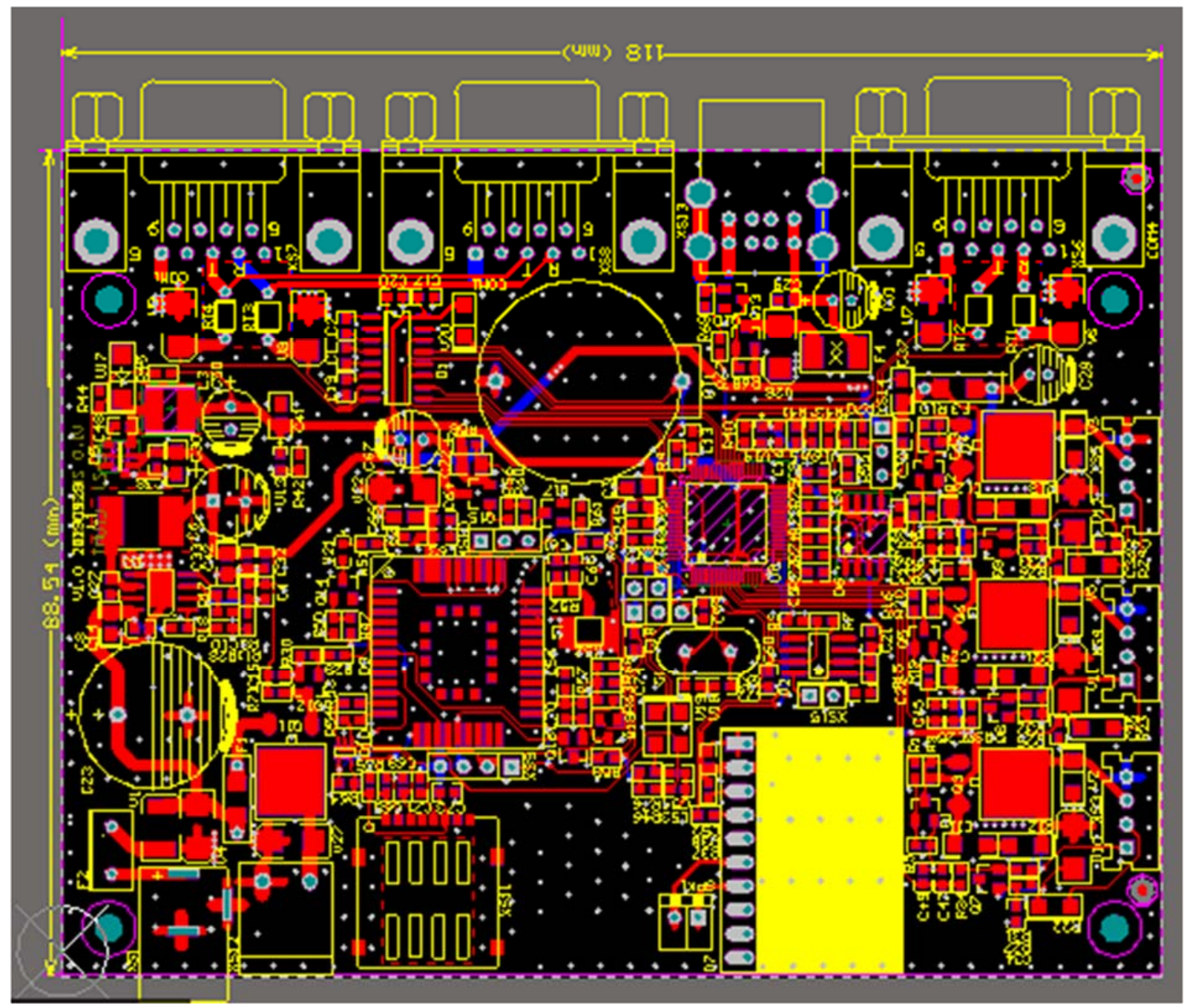

Fig.5 PCB diagram of the circuit

\section{Conclusion}

The identification and printing module uses NB-IoT technology, it makes full use of NB-IoT's characteristics of wide coverage, large capacity, low cost and low power consumption to monitor the status of garbage collection equipment at any time. At the same time, it has voice broadcast function, which can remind users to carry out corresponding operation in time. The recyclable categories of garbage collection system after applying the identification and printing module are not limited to the existing carton and gold Plastic, etc., can be more categories, can greatly achieve the recycling rate of recyclable items, at the same time save labor, do not need to wait for special personnel to recycle, has high promotion and application value.

\section{References}

1. Jiayu Tian, Qingru Song, Yao Wang. Based on the Internet of things residential garbage sorting and recycling system $[\mathrm{J}]$. Computer knowledge and technology, 13, 245-246 (2017).
2. Rui Sun, Kuntai Meng, Yu Ji, et al. Research on intelligent supervision system of garbage collection equipment based on NB-IoT [J]. Design and application of communication, 2,125-126(2020).

3. Tong Wu. Research on garbage sorting and recycling system based on artificial intelligence technology [J], Science and Technology \& Innovation, 9,38-39 (2020).

4. LIU Guangkuo, SHI Yanqing, LIU Xi-xing. Intelligent recycling management system for domestic garbage based on internet of things technology[J]. Journal of the Hebei Academy of Sciences,36,13-16, (2019).

5. Qing Su, Jieling Deng. "Cloud bucket" -Application of Intelligent Cloud garbage collection system[J]. Construction engineering technology and design,11,3599(2018). 